\title{
The discrete Radon transform: A more efficient approach to image reconstruction?
}

\author{
Andrew Kingston ${ }^{a}$, Imants Svalbe ${ }^{b}$, and Jean-Pierre Guédon ${ }^{c}$ \\ ${ }^{a}$ Dept. Applied Math. RSPhysSE, The Australian National University, Canberra 0200, \\ Australia; \\ ${ }^{b}$ School of Physics, Monash University, Clayton 3800, Australia; \\ ${ }^{c}$ IRCCyN-IVC, École polytech., l'Université de Nantes, Rue Christian Pauc, La Chantrerie \\ BP50609 Cedex 3, France.
}

\begin{abstract}
The Radon transform and its inversion are the mathematical keys that enable tomography. Radon transforms are defined for continuous objects with continuous projections at all angles in $[0, \pi)$. In practice, however, we pre-filter discrete projections taken at a discrete set of angles and reconstruct a discrete object. Since we are approximating a continuous transform, it would seem that acquiring more projections at finer projection resolutions is the path to providing better reconstructions. Alternatively, a discrete Radon transform (DRT) and its inversion can be implemented. Then the angle set and the projection resolution are discrete having been predefined by the required resolution of the tomogram. DRT projections are not necessarily evenly spaced in $[0, \pi)$, but are concentrated in directions which require more information due to the discrete square [or cubic] grid of the reconstruction space. A DRT, by design, removes the need for interpolation, speeding up the reconstruction process and gives the minimum number of projections required, reducing the acquisition time and minimizing the required radiation dose. This paper reviews the concept of a DRT and demonstrates how they can be used to reconstruct objects from X-ray projections more efficiently in terms of the number of projections and to enable speedier reconstruction. This idea has been studied as early as 1977 by Myron Katz. The work begun by Katz has continued and many methods using different DRT versions have been proposed for tomographic image reconstruction. Here, results using several of the prominent DRT formalisms are included to demonstrate the different techniques involved. The quality and artifact structure of the reconstructed images are compared and contrasted with that obtained using standard filtered back projection.
\end{abstract}

Keywords: discrete Radon transform, tomographic reconstruction

\section{INTRODUCTION}

The Radon transform (RT) and its inversion are the mathematical keys that enable tomography. The acquisition of X-ray projections of an object is effectively the RT of the density function for that object. The recovery of this density function is achieved by applying some form of inverse RT to the projection data.

It was Austrian mathematician Johann Radon who, in $1917^{*}$, first discovered that a continuous function, $f(x, y)$, can be recovered from its infinite set of projections, $p_{f}(\rho, \theta), \theta \in[0, \pi)$ as:

$$
f(x, y)=\mathcal{R}^{\dagger} \mathcal{K} \mathcal{R} f(x, y)=\mathcal{R}^{\dagger} \mathcal{K} p_{f}(\rho, \theta)
$$

where $\mathcal{R}$ denotes the Radon projection operator defined as

$$
\mathcal{R} f(x, y)=\int_{-\infty}^{\infty} \int_{-\infty}^{\infty} f(x, y) \delta(\rho-x \sin \theta+y \cos \theta) \mathrm{d} x \mathrm{~d} y=p_{f}(\rho, \theta),
$$

Further author information: (Send correspondence to AK)

E-mail: andrew.kingston@anu.edu.au, imants.svalbe@sci.monash.edu.au, jean-pierre.guedon@univ-nantes.fr

*An english translation of Radon's original paper can be found in ${ }^{1}$

Developments in X-Ray Tomography VI, edited by Stuart R. Stock,

Proc. of SPIE Vol. 7078, 70780G, (2008) $\cdot 0277-786$ X/08/\$18 $\cdot$ doi: 10.1117/12.795779

Proc. of SPIE Vol. 7078 70780G-1

Downloaded from SPIE Digital Library on 26 Apr 2010 to 150.203.243.22. Terms of Use: http://spiedl.org/terms 
$\mathcal{K}$ denotes the ramp filter convolution operator defined as

$$
\mathcal{K} p_{f}(\rho, \theta)=\mathcal{F}_{1 D}^{-1}\{|\omega|\} * p_{f}(\rho, \theta)=\int_{-\infty}^{\infty}|\omega| \widehat{p}_{f}(\omega, \theta) \exp [i 2 \pi \rho \omega] \mathrm{d} \omega=\widetilde{p}_{f}(\rho, \theta),
$$

where $\widehat{p}_{f}(\omega, \theta)$ is the $1 \mathrm{D}$ Fourier transform of $p_{f}(\rho, \theta)$, i.e., $\widehat{p}_{f}(\omega, \theta)=\mathcal{F}_{1 D}^{+1} p_{f}(\rho, \theta)=\int_{-\infty}^{\infty} p_{f}(\rho, \theta) \exp [-i 2 \pi \omega \rho] \mathrm{d} \rho$, and $\mathcal{R}^{\dagger}$ denotes the dual of the projection operator (known as the back-projection operator) defined as

$$
\mathcal{R}^{\dagger} \widetilde{p}_{f}(\rho, \theta)=\int_{0}^{\pi} \widetilde{p}_{f}(\rho, \theta) \mathrm{d} \theta=f(x, y) .
$$

For the continuous case, the inverse RT is ill-posed and a unique solution only exists if the set of projections is infinite. In practice, however, only a finite approximation of $f$ at some given resolution is required. Therefore, a finite set of discretely sampled projections is sufficient for reconstruction using a discretisation Radon's filtered back-projection (FBP) formula. FBP has become the standard inversion technique, as it is regarded to be less sensitive to interpolation errors than techniques based in the frequency domain, i.e., Fourier inversion (FI). FBP has been used relatively unaltered for the last 40 years, with limited attention to the area of filter design.

The standard data acquisition procedure, i.e., to obtain a set of projections equi-spaced in angle, gives a discrete sampling of the RT of the continuous object/function in the form of a polar tiling (in both Radon and Fourier space) which has no straight-forward inversion to a discrete square [or cubic] image array. This leads to arbitrary filtering and/or interpolation in the reconstruction process, which, in turn, produces image reconstruction artifacts.

This paper seeks to review and explore methods to improve both the implementation and results of reconstruction by taking into account that the solution required is not $f$ but the digital image of $f$. Beylkin ${ }^{2}$ in his original DRT paper showed that for an exactly invertible discrete reconstruction, the inversion, whilst similar to a discretisation of Radon's filtered back-projection (FBP) formula, cannot be directly derived from this. Discretising the projections and filter according to the image grid, as proposed by Guédon and Bizais ${ }^{3}$ and Horbelt et $a l,{ }^{4}$ can improve the quality of reconstructed images significantly and reduce the total number of projections required. They showed that when a square pixel model is assumed, the appropriate discrete filter varies for each projection angle. Another aspect that has been relatively unexplored is the projection angle set. If the filter is not symmetrical for a square grid then surely the projection angle density required also varies with angle. Katz ${ }^{5}$ has investigated this idea with some interesting results.

The work of Horbelt et al was notable for showing that the use of discrete grid-related filters (e.g. Bsplines) dramatically improves the quality of the reconstructions in the least squares sense and reduces the level of image artifacts. This approach enabled the same quality reconstructions to be obtained using either fewer interpolations, or using a smaller number of projection angles.

Many DRT formalisms exist ${ }^{2,6-9}$ which are fast and exactly invertible mappings from/to a square image array. If the projection data can be acquired to map onto one of these tilings of Radon space, or can be easily arrived at from a polar tiling with minimal interpolation, then the process of inversion from this point is trivial. Each DRT formalism has the potential to be a more efficient tool for reconstruction in one or more of several ways:

1 - It should be faster, since, by design, it requires trivial or no interpolation once the projection data has been mapped to the required format. Also, since mapping from projection to image space is lossless and fast, iterative improvements (such as the removal of beam hardening artifacts) should be simple and fast operations.

2 - It should produce tomograms of equivalent quality from fewer projections compared with standard FBP, since its projection set should (in the process of defining the DRT) be optimised for a square grid. For example Katz $^{5}$ achieved this by assuming a digital algebraic reconstruction and determining the maximum sized image space that allowed a unique reconstruction. These discrete projections are not usually equi-spaced in angle. Since only a digital image of $f$ is desired, the number of reconstructed levels (i.e., the grey-scale resolution) in the image can also often be pre-determined, which can further reduce the number of projections required. 
3 - It should be more accurate, since no arbitrary filtering is required and interpolation of the data can be minimised.

The paper is organised as follows: Section 2 gives a broad overview of some of the DRT formalisms developed to date. The three following sections discuss how DRT formalisms can fine-tune reconstructions, as mentioned above: Section 3 - minimising angle sets, Section 4 - minimising interpolation, and Section 5 - minimising computation time. Apart from outlining some DRT definitions, a study of angle selection by M. Katz ${ }^{5}$ is outlined, along with an improved method to discretise the projections and the filter for FBP, as developed by Guédon and Bizais. ${ }^{3}$ Finally the performance of each method is compared and contrasted in Section 6 , before making some concluding remarks in Section 7.

\section{DISCRETE RADON TRANSFORM FORMALISMS}

The are several desirable properties that a DRT formalism should include: it should provide an exactly invertible mapping from image space to projection space, this mapping should be fast. If possible, the Fourier slice theorem and hence the convolution property [which are both properties of the continuous RT] should hold. This section outlines several DRT formalisms that satisfy all of the above properties. A comprehensive review of DRT formalisms is given in. ${ }^{10}$ As the DRT has no $1 \mathrm{D}$ analogue, it cannot be derived as a trivial extension from 1D, as is the case for the discrete Fourier transform (DFT). The essential difference between each DRT approach lies in the detailed description of how to define a line on a square image grid.

\subsection{Finite Radon transform (FRT) and Mojette transform}

The first type defines a discrete line as the set of pixels (not necessarily connected) centered on a "rational" line. Here rational implies the slope, $m$, can be found as the inverse tangent of an irreducible rational fraction [also known as a Farey fraction], see Fig. 6-right. Line integrals are simply the sum of the values of all pixels taken along line trajectories in the set. This assumes the Dirac pixel model, i.e., that each pixel occupies an infinitely small point in the image space. Other models that include projected contributions from adjacent pixels are exemplified by the Haar pixel model. Higher order Haar models can be achieved by simply convolving the Dirac model projections with the projection of the pixel model at the desired order.

There are two main methods using this line definition; the $\mathrm{FRT}^{6}$ and Mojette transform, ${ }^{7}$ both transforms are similar. The principal differences are that the FRT assumes the image is periodic in both the $x$ and $y$ directions, (i.e., toroidal, as for the DFT), and is defined over $p \times p$ arrays where $p$ is prime. A review of FRT properties and applications is given in. ${ }^{10}$ The angle sets of both DRTs belong to the set of Farey angles, $\theta=\tan ^{-1}(a / b)$ where $\operatorname{gcd}(a, b)=1$ and the projection sampling rate varies as $\sqrt{a^{2}+b^{2}}$.

The FRT has a pre-defined set of $p+1$ projections as depicted in Fig. 1 for $p=5$. Computation of its inverse is very similar to the projection process and is well-conditioned. Both projection and reconstruction can also be performed in the frequency domain.
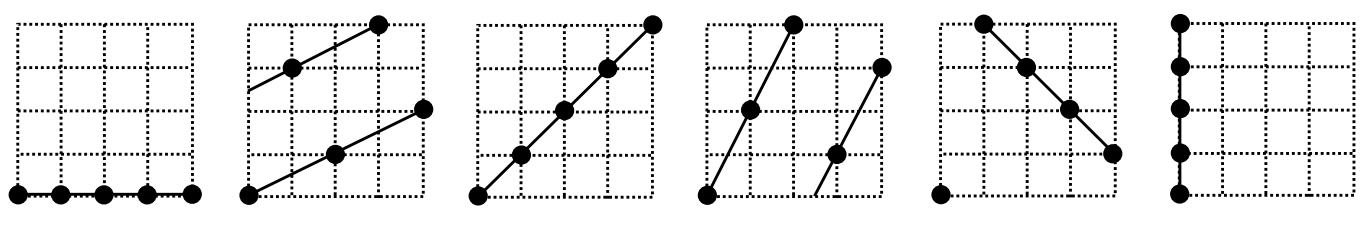

Figure 1. The six FRT discrete lines through the origin of a $5 \times 5$ grid.

The Mojette transform has an arbitrary set of rational projection angles (some examples of Mojette lines are given in Fig. 2) and is uniquely reconstructible provided the Katz ${ }^{5}$ criterion is satisfied. Various reconstruction methods have been developed, ranging from fast, exact, but ill-conditioned methods, ${ }^{7}$ to robust iterative algebraic solutions. $^{11}$ 


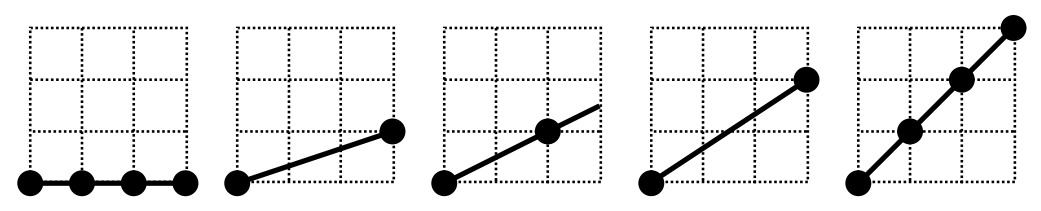

Figure 2. Some Mojette discrete lines through the origin of a $4 \times 4$ grid with slopes, $m \in[0,1]$.

\subsection{Linogram or fast slant stack (FSS)}

A second type divides Radon space into two categories, vertical lines $(-1 \leq-1 / m<1)$ and horizontal lines $(-1 \leq$ $m<1)$. Vertical lines sample each image row once, however may not have an integer column value [and like-wise for horizontal lines]. Vertical [resp. horizontal] line integrals are found as the sum of the trigonometrically interpolated values of adjacent pixels in each row [resp. columns], see Fig. 3. The interpolation is performed in the frequency domain using the $1 \mathrm{D}$ Chirp-Z transform. The angle set is comprised of equi-spaced slopes with $m=2 l / N$ for $l \in[-N / 2, N / 2-1]$ for an $N \times N$ image (see Fig. 6-center) and produces a discrete sampling of Radon space in the form of concentric squares [termed pseudo-polar], see Fig. 7-center. A fast and exact iterative inverse was developed by Averbuch et al, ${ }^{8}$ where the inversion is performed in the frequency domain.
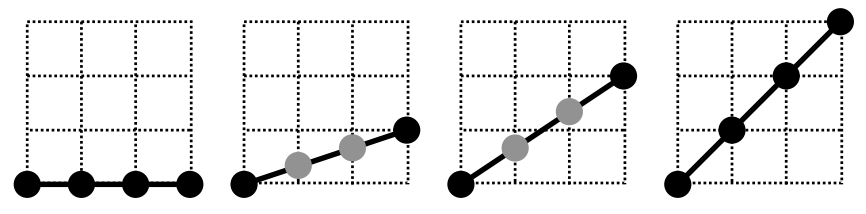

Figure 3. The four equi-spaced slope discrete lines through the origin of a $4 \times 4$ grid with slopes $m \in[0,1]$. Note that intermediate grey pixels are found through $1 \mathrm{D}$ trigonometric interpolation.

\subsection{Approximate discrete Radon transform (ADRT)}

This third type can be considered similar to the FSS but "snaps" to whole pixels in each row for vertical lines (or column for horizontal lines), generating sets of 8-connected pixels that closely approximate continuous lines, see Fig. 4. These connected sets (or discrete lines) are termed d-lines. This approach was developed by Götz and Drückmuller ${ }^{12}$ and later, independently, by Brady. ${ }^{13}$ The set of pixels belonging to a line are not selected as those closest to the line, but as those that enable a fast, $\mathrm{O}\left(N^{2} \log N\right)$ for an $N \times N$ image, projection using a method similar to that in the fast DFT (FFT). An upper limit to the distance from a continuous line a pixel lies is $0.5(\log N-1){ }^{13}$ The angle set is also equi-spaced in slope as for the FSS. A fast and exact, multi-resolutional, iterative inverse was developed by Press, ${ }^{9}$ the complexity of which is also $\mathrm{O}\left(N^{2} \log N\right)$.
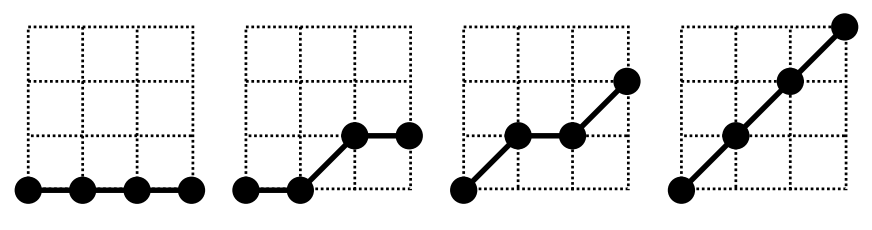

Figure 4. The four 8-connected discrete lines (d-lines) through the origin of a $4 \times 4$ grid with average slopes $m \in[0,1]$.

\section{MINIMISING THE NUMBER OF PROJECTIONS}

Guédon and Bizais ${ }^{3}$ and Horbelt et $a l^{4}$ have developed a more appropriate method to discretise filtered back projection. This approach gives higher quality reconstructions and ultimately requires fewer projections than standard filtered back-projection. Since a square pixel (assuming the Haar model as described below) is not radially symmetric, the contribution each pixel value makes to a projection varies with the projection angle. A discrete filter that accommodates this variation with angle is to be prefered over uniform filters. 
In practice, the function required to be reconstructed is not $f(x, y)$, but a discretised version of $f$ which can be found as the $2 \mathrm{D}$ convolution of $f$ with a pixel intensity distribution model, $b(x, y)$, i.e., $F[k, l]=b(x, y) *$ $\left.* f(x, y)\right|_{x=k, y=l}$. The convolution property gives $\mathcal{R}\{b(x, y) * * f(x, y)\}=p_{b}(\rho, \theta) * p_{f}(\rho, \theta)$ so Eq. (1) becomes:

$$
b(x, y) * * f(x, y)=\mathcal{R}^{\dagger} \mathcal{K}\left\{p_{b}(\rho, \theta) * p_{f}(\rho, \theta)\right\}=\mathcal{R}^{\dagger}\left\{k_{b}(\rho, \theta) * p_{f}(\rho, \theta)\right\},
$$

where $k_{b}(\rho, \theta)=\mathcal{F}_{1 D}^{-1}\left\{|\omega| \widehat{p}_{b}(\omega, \theta)\right\}$. A useful pixel model that is commensurate with discrete display devices is the separable Haar basis defined as:

$$
s(x-k, y-l)=s(x-k) s(y-l), \quad \text { where } \quad s(z)=\left\{\begin{array}{ll}
1, & |z|<\frac{1}{2} \\
\frac{1}{2}, & |z|=\frac{1}{2} \\
0, & |z|>\frac{1}{2}
\end{array} .\right.
$$

Figure 5 gives a depiction of the Haar pixel model and the the variation in projection contribution depending on angle.

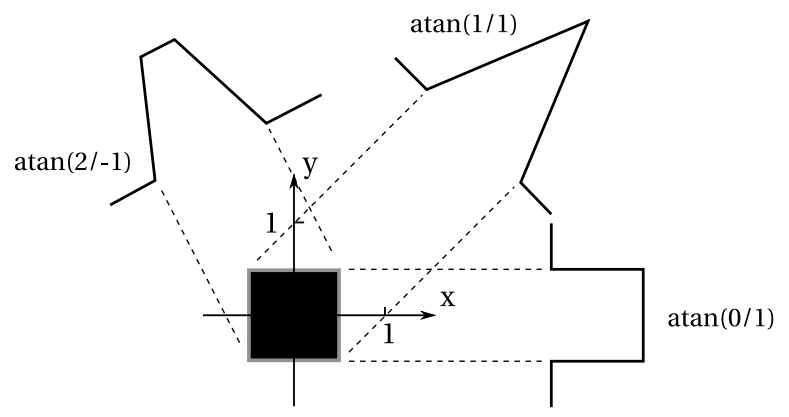

Figure 5. The Haar pixel model with three projections at the "rational" or Farey angles, $\tan ^{-1}(-2 / 1), \tan ^{-1}(1 / 1)$, and $\tan ^{-1}(0 / 1)$.

Guédon and Bizais ${ }^{3}$ give analytical solutions to the Haar filter functions $k_{s}(\rho, \theta)$ which vary with projection angle $\theta$ and incorporate an oversampling rate $r$ for $r>1$. They compared the result obtained from standard FBP with that of this Haar-FBP with $r=4$. They showed that using the angle varying Haar filter suppresses the line artifacts that are evident using the standard FBP and in fact showed that the only artifacts were those arising from the limited frequency bandwidth. Horbelt et al ${ }^{4}$ provide a similar result using spline convolutions. They find that better quality reconstructions (in terms of PSNR) arise by doubling the sampling rate in the projections rather than by doubling the number of projection angles.

Another method to minimise the number of projections required is to select projection angles that maximise the information according to the pixel model of the reconstruction. This was explored in depth by Katz ${ }^{5}$ in the 1970s. He assumes inversion using the algebraic reconstruction technique (ART) and shows that the optimal angles to use, assuming the Haar pixel model, are the "rational" or "Farey" angles found as the inverse tangent of irreducibe fractions, i.e., $\theta=\tan ^{-1}(a / b)$ where $\operatorname{gcd}(a, b)=1$, see Fig. 6 -right. The projections at these angles minimise the "mixing" of pixel contributions, thus minimising the required resolution of the projections. The use of Farey angles enables the construction of discrete ghost functions. These are functions that exist in image space but project to zero at the specified projection angle set. The finest resolution of a reconstruction, $M \times N$, that cannot contain a ghost function (i.e., the support of the discrete ghost function is larger than $M \times N$ ) is the finest reconstruction resolution that gives a unique solution.

Katz gives an example in ${ }^{5}$ assuming 36 projections. Standard FBP with equi-spaced projection angles, using basic sampling theory $N=2 P / \pi=72 / \pi \approx 23$, a $23 \times 23$ image can be reconstructed. Katz selects 36 Farey fractions that all lie within $1.5^{\circ}$ of the 36 equi-spaced projection angles and the smallest discrete ghost function has support of $94 \times 94$ pixels, thus a $93 \times 93$ image can be reconstructed uniquely, at approximately 4 times finer resolution than using standard FBP.

The Katz angle selection method, based on ART, would benefit further by Haar filtering the projections, i.e., performing a convolution with $p_{s}(\rho, \theta)$. This idea has been investigated by Servieres et al ${ }^{11}$ using the Mojette transform with ART inversion using the conjugate gradient method (CGM). 

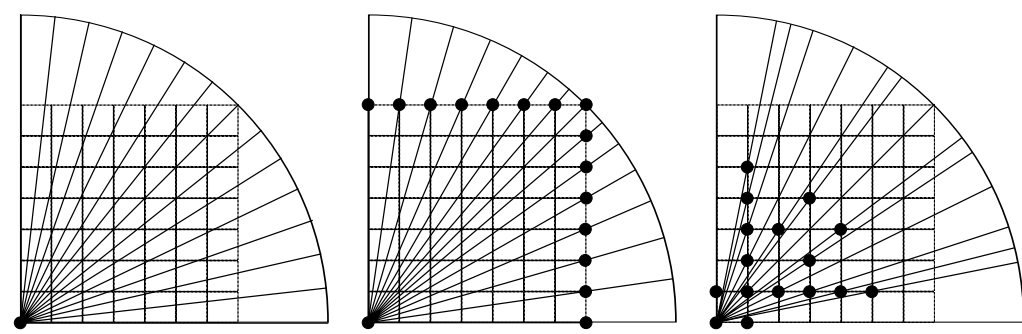

Figure 6. Three types of angle distributions used: left: equi-spaced in angle, center: equi-spaced in slope, right: "rational" or Farey angles.

A further possibilty to minimise the number of projections is to employ the FRT. This is not an algebraic approach, but by design is a well-conditioned back projection method that requires no filtering. Of all the DRT formalisms this is the only non-redundant version and so must be optimal. For a $p \times p$ image [where $p$ is prime], only $p+1$ projections with $p$ samples are required. Svalbe et al in ${ }^{14-16}$ have developed many methods to map sampled continuous projection data to the discrete projections of the FRT; this is the only step in the inversion process where errors are introduced. The incorporation of the Haar filter to pre-condition the real projection data values $^{3,4}$ is an avenue which has not yet been fully explored.

A final method which may be employed to minimise the number of projections for an object comprised of 1 or more regions of uniform material is to make use of discretised intensity levels. For example, an object consisting of a single material, e.g., an aluminium foam, only requires a binary reconstruction with 0 indicating air and 1 indicating a pixel containing the object. This drastically reduces the amount of information required. ${ }^{17,18} \mathrm{In}$ fact for certain classes of shapes, e.g., hv-convex, only 2 projections [row and column sums] are required for a unique reconstruction. ${ }^{19}$

\section{MINIMISING INTERPOLATION}

All Radon inversion methods require some form of interpolation. The Fourier inversion (FI) technique requires interpolation from the polar grid (Fig. 7-left) of collected data to a square grid (Fig. 7-right) in the frequency domain. The filtered back-projection (FBP) technique requires interpolation for every pixel in the image for every projection, i.e., $P N^{2}$ interpolations. This interpolation in the spatial domain appears to be much less sensitive than in the frequency domain as used for FI.

The FSS and ADRT both use equi-spaced slope projections, forming a set of concentric squares [termed pseudo-polar], as shown in Fig. 7-center. The projection data from these transforms have a much smaller interpolation distance from the standard polar projection data than directly to a square grid as for FI, and is performed in the projection domain [Radon space]. It serves as an intermediate step in mapping from the polar grid of projection data to the square image grid. As with FI, after this initial interpolation, no further interpolation is required. The projections could be purposefully acquired at equi-spaced slopes to further reduce the need for data interpolation (only 1D in this case).
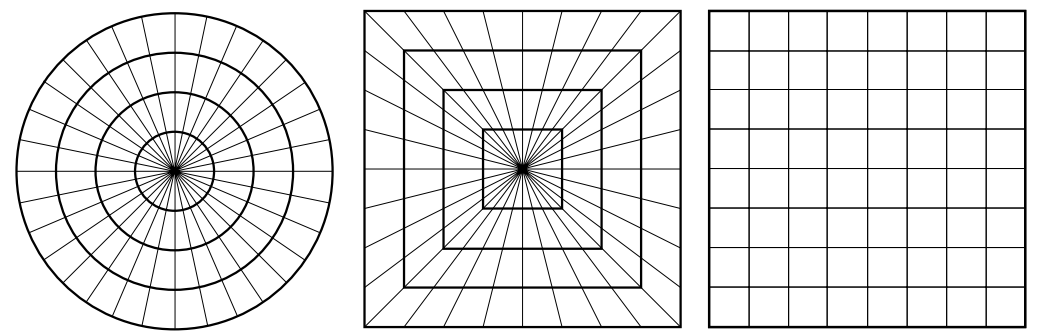

Figure 7. Three types of discrete grids used: left: polar (concentric circles), center: pseudo-polar (concentric squares), right: square. 
Mojette and FRT back-projection use no interpolation, owing to the use of Farey angles and the Dirac pixel model. Assuming the Haar pixel model, interpolation is required in the projections, performed as a convolution with the projection of a Haar pixel. Since all pixels centered on a line at angle $\theta$ each have the same interpolation values, this is done just once in the projection as back projection requires no interpolation.

\section{MINIMISING COMPUTATION TIME}

By design, DRT formalisms are very efficient methods for projection and reconstruction on a discrete square grid. Standard FBP of an $N \times N$ image with a number of projections, $P \approx \pi N / 2$, requires $\mathrm{O}\left(N^{3}\right)$ operations [or $\mathrm{O}\left(N^{4}\right)$ for $3 \mathrm{D}$ reconstructions].

The Linogram or FSS is computed via the frequency domain using a series 1D Chirp-Z and 1D FFT operations and can be computed in $\mathrm{O}\left(N^{2} \log N\right)$ operations [or $\mathrm{O}\left(N^{3} \log N\right)$ for 3D reconstructions]. For a $2048^{3}$ image, reconstruction via FSS should be approximately 200 times faster.

In the set of digital lines defined for the ADRT, pixels are not selected as the nearest grid points to continuous lines, which would minimise discretisation error, but those that can be computed efficiently in $\mathrm{O}\left(N^{2} \log N\right)$ operations. This is not a fast DFT (FFT) method, but exploits a similar concept [i.e., butterfly operations] applied directly to sub-image sums. How well these d-lines approximate continuous lines is analysed by finding an upper limit to the distance perpendicular to the line from a given pixel belonging to the d-line. Götz and Drückmuller show that this is $\mathrm{O}(\log N)$, while Brady further refines this to $0.5(\log N-1)$.

The FRT can be computed exactly by both direct back-projection (BP) in $\mathrm{O}\left(p^{3}\right)$ operations and $\mathrm{FI}$ in $\mathrm{O}\left(p^{2} \log p\right)$ operations. However, BP requires no interpolation and can be computed extremely efficiently using a block copy and add routine and is in fact faster than FI for $p<800$.

To date the Mojette transform has no fast well-conditioned inversion. The search for a pre-conditioner continues and a multi-scale Mojette transform has been developed ${ }^{20}$ which may enable fast reconstruction using a multi-grid method similar to that developed by Press for the ADRT.

\section{TOMOGRAPHIC RECONSTRUCTION VIA DISCRETE RADON TRANSFORMS}

This section is included to demonstrate the tomographic reconstruction performance of several of the DRT formalisms as compared with standard Ram-Lak filtered back-projection.

\subsection{Results}

Figure 8 depicts four $512 \times 512$ reconstructions of the slice of a rock core each using a different inversion technique. The sinogram (which has 180 projections equi-spaced in angle each with 682 ray-sums) has been interpolated from a fan beam sinogram. This is purposely insufficient information in order to emphasize the artifacts introduced by each method. Figure $8 \mathrm{a}$ is the result of FBP which should require over projections, $8 \mathrm{~b}$ is the results using FRT (as outlined $\mathrm{in}^{16}$ ) which should require at least 522 projections (nearest prime is 521 ), $8 \mathrm{c}$ is the result using FSS which should require 1024 projections, and 8d is the result using ADRT which should require 2048 projections. A $100 \times 100$ zoomed window of each reconstruction is provided in Fig. 9 to compare image quality. No manipulation of data or reconstructed images was performed (except scaling grey levels), the required DRT projection data was simply interpolated to the required angle sets and inserted directly into the DRT projections for inversion.

\subsection{Discussion}

It can be seen from the results that FBP has given possibly the sharpest image but despite the angle set being ideally suited for FBP, it is the noisiest and has the most prominent artifacts. The DRT methods have given far smoother data with much less significant artifacts. The FRT, FSS, and ADRT require an increasing number of projections respectively and use very different inversion methods but have all produced comparable images. The images appear sharp towards the center but increasingly blurry towards the extremities. This is possibly since interpolation from fan beam to parallel beam is accurate around origin and increasingly blurred with radius (as the fan beams deviation from parallel increases with the square of the distance from the origin). The reconstructions

Proc. of SPIE Vol. 7078 70780G-7 
(a)

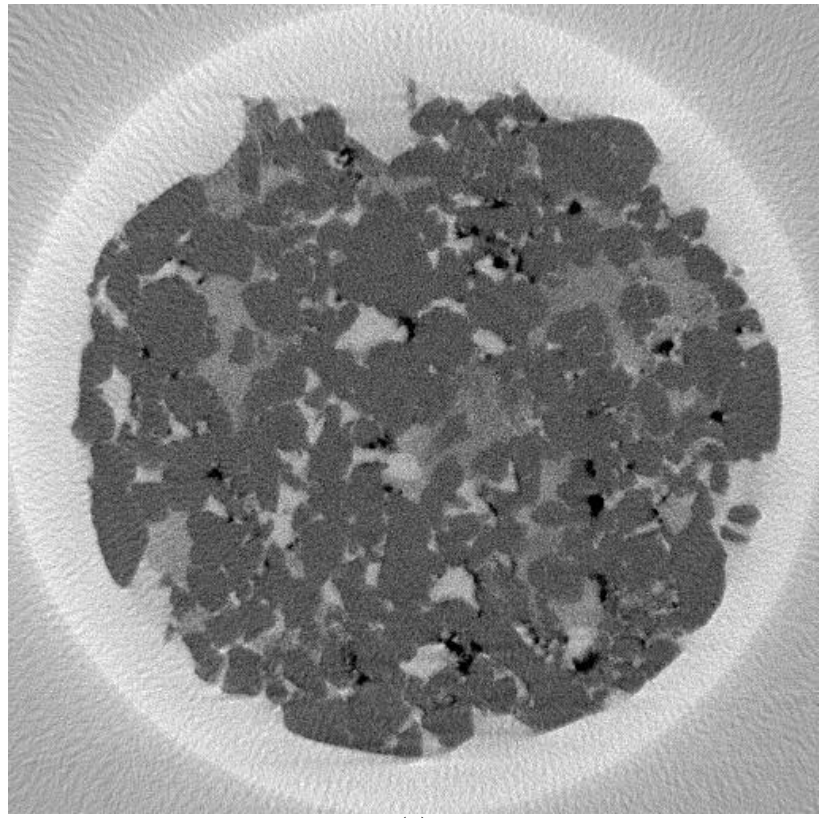

(c)

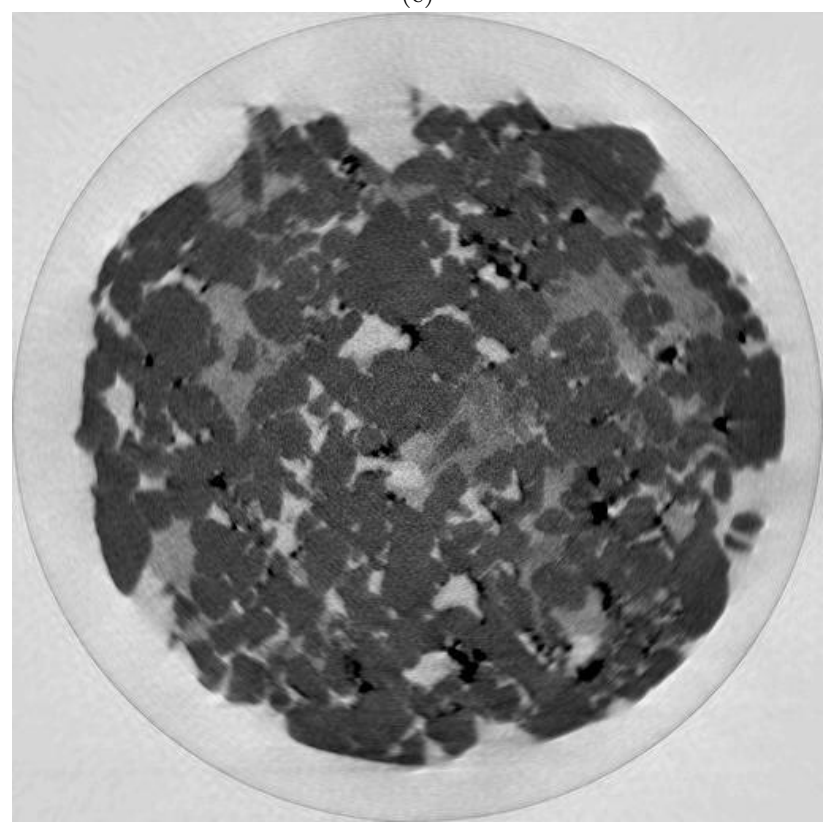

(b)

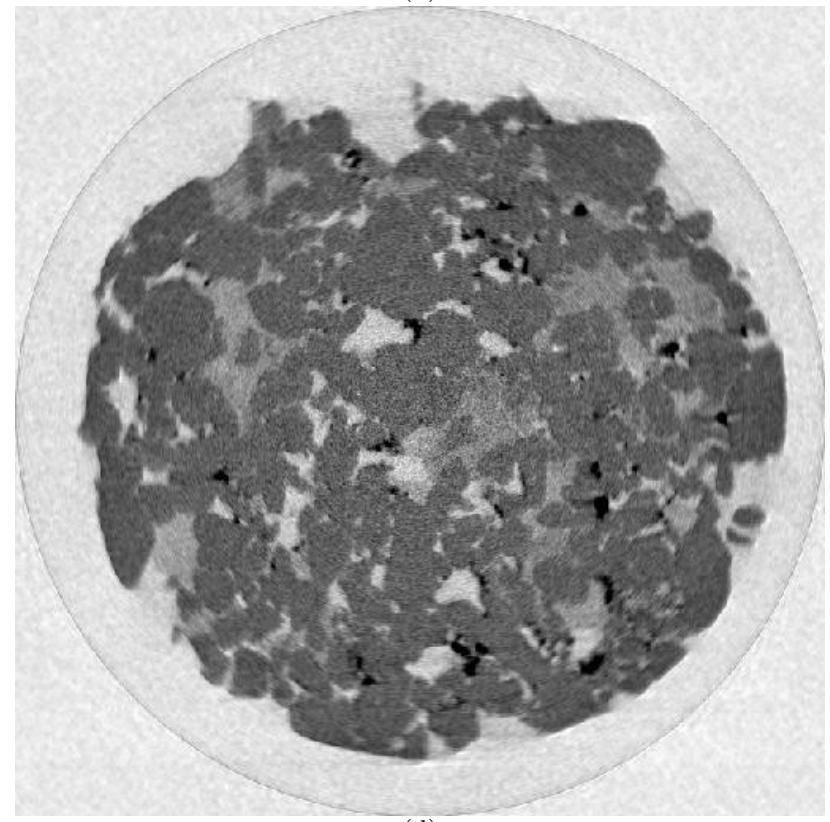

(d)

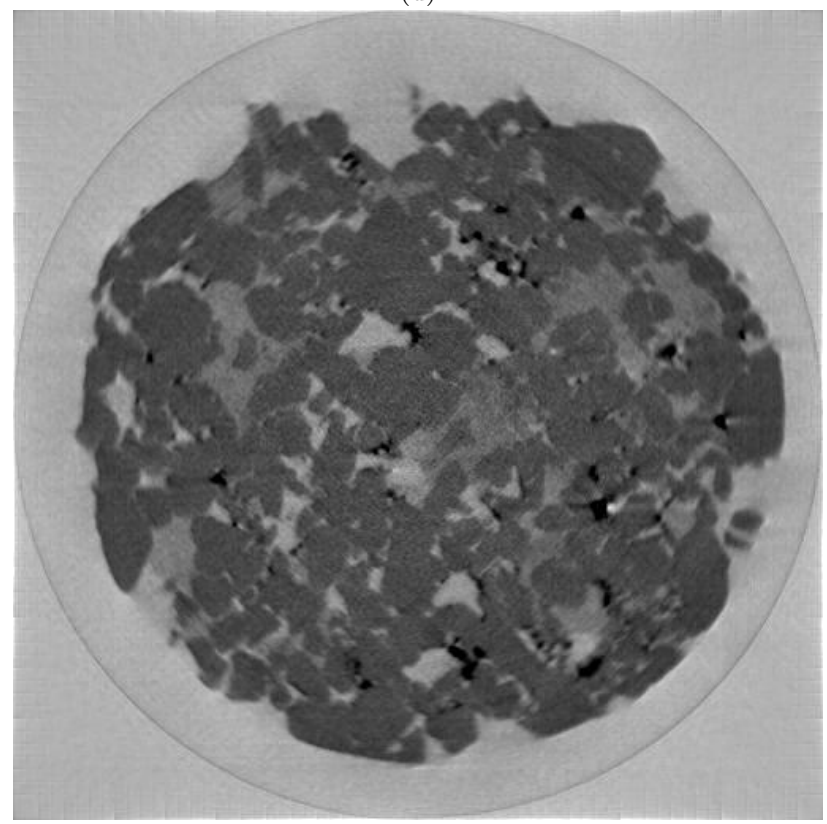

Figure 8. $512 \times 512$ reconstructions from a $683 \times 180$ sinogram using: a) FBP, b) FRT, c) FSS, d) ADRT.

using FSS ad ADRT appear very similar. This is most likely since they both utilise the same angle distribution. The artifact structures are slightly different owing to the different inversion procedures ( "streaking" for the back-projecting FSS and "blockiness" for the multi-scale ADRT) but otherwise the reconstructed images have very similar properties. The FRT has its own unique artifacts: "blotchiness". This could be due to the angular interpolation required to map to the $p+1$ Farey angles which are not necessarily equally spaced. There may be densely sampled angular regions from the FRT in which all projection data is interpolated from a common pair of nearest angle acquired projections. Ideally, projections acquired to match the DRT angle set of each reconstruction method would be give a better comparison. 
(a)

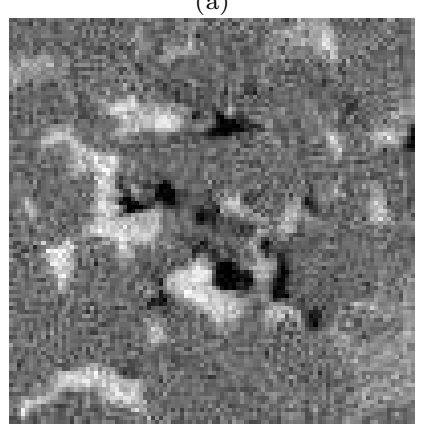

(b)

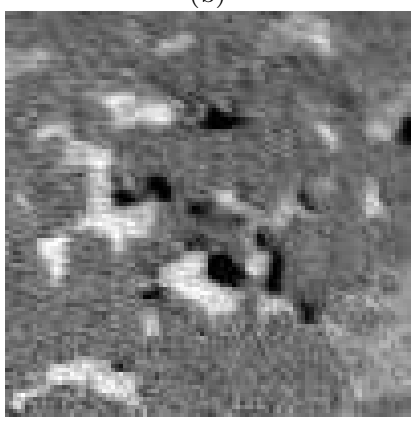

(c)

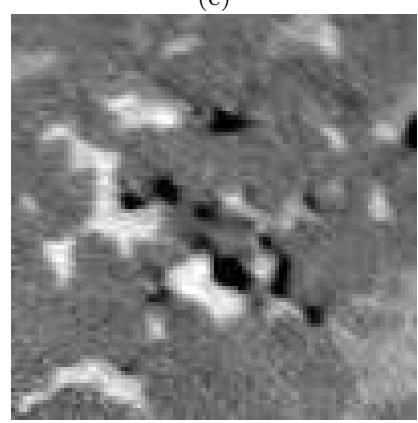

$(\mathrm{d})$

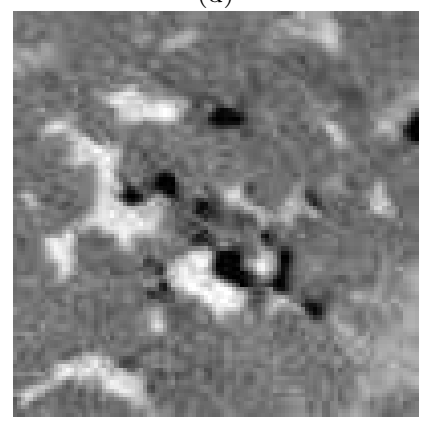

Figure 9. $100 \times 100$ zoomed region of the reconstructions in Fig. 8: a) FBP, b) FRT, c) FSS, d) ADRT.

\section{CONCLUSIONS}

The purpose of the paper is to expose the X-ray tomography community to the concept of discrete Radon transforms and the idea of concentrating on reconstructing a digital image of $f$ (as opposed to $f$ itself). With this in mind the sampled continuous projections of $f$ should be filtered according to the pixel model being used for the reconstructed image, commonly the Haar pixel model. This removes image reconstruction artifacts. The projection angle set should not necessarily be equi-spaced in angle but vary according to digital orientation of $x, y, z$-axes and the reconstruction method/DRT used. DRT formalisms, by design, are better suited to reconstructing digital images. They provide faster reconstruction with less/no interpolation and no arbitrary filtering. Mapping acquired projection data to the form required by the DRT projections is the only step where errors are introduced. Projections acquired to best match these forms, (as equi-spaced angle sets best suits FBP), is the ideal situation.

\section{REFERENCES}

[1] Deans, S., [The Radon Transform and Some of Its Applications, revised edition], Krieger, Malabar, FL (1993).

[2] Beylkin, G., "Discrete Radon Transform," IEEE Trans. Acoustics, Speech and Sig. Processing 35(2), 162172 (1987).

[3] Guédon, J. and Bizais, Y., "Bandlimited and Haar filtered back-projection reconstructions," IEEE Trans Med Imaging 13(3), 430-40 (1994).

[4] Horbelt, S., Liebling, M., and Unser, M., "Discretization of the Radon transform and of its inverse by spline convolutions," IEEE Trans Med Imaging 21(4), 363-76 (2002).

[5] Katz, M., [Questions of uniqueness and resolution in reconstruction from projections], Lect. Notes in Biomath., Springer Verlag (1977).

[6] Matúš, F. and Flusser, J., "Image representation via a finite Radon transform," IEEE Transactions on Pattern Analysis 83 Machine Intelligence 15(10), 996-1006 (1993).

[7] Guédon, J. and Normand, N., "The Mojette transform: applications in image analysis and coding," The International Society for Optical Engineering 3024(2), 873-884 (1997).

[8] Averbuch, A., Coifman, R., Donoho, D., Israeli, M., and Walden, J., "Fast slant stack: a notion of Radon transform for data in a Cartesian grid which is rapidly computible, algebraically exact, geometrically faithful and invertible," www-stat.stanford.edu/ donoho/Reports/2001/FastSlantStack.pdf (preprint 2001).

[9] Press, W., "Discrete Radon transform has an exact, fast inverse and generalizes to operations other than sums along lines," Proc. Nat. Acad. Sci. USA. 103, 19249-54 (2006).

[10] Kingston, A. and Svalbe, I., "Projective transforms on periodic discrete image arrays," P. Hawkes (Ed), Advances in Imaging and Electron Physics 139 (2006).

[11] Servières, M., Idier, J., Normand, N., and Guédon, J., "Conjugate gradient Mojette reconstruction," in [Proc. SPIE Medical Imaging 2005: Image Processing], Fitzpatrick, J. and Reinhardt, J., eds., 5747, 206774 (2005). 
[12] Götz, W. and Druckmüller, H., "A fast digital Radon transform ? an efficient means for evaluating the Hough transform," Pattern Recognition 29, 711-718 (1996).

[13] Brady, M., "A fast discrete approximation algorithm for the Radon transform," SIAM Journal on Computing 27, 107-119 (1998).

[14] Svalbe, I. and van der Spek, D., "Reconstruction of tomographic images using analog projections and the digital Radon transform," Linear Algebra and Its Applications 339, 125-145 (Dec 2001).

[15] Svalbe, I. and Kingston, A., "Intertwined digital rays in discrete Radon projections pooled over adjacent prime sized arrays," in [Proceedings of 11th International Conference Discrete Geometry for Computer Imagery], 2886, 485-494 (2003).

[16] Kingston, A. and Svalbe, I., "Mapping between digital and continuous projections via the discrete Radon transform in Fourier space," in [Proc. 7th International Conference on Digital Image Computing: Techniques and Applications], 263-272, CSIRO Publishing, Sydney, Australia (2003).

[17] Batenburg, K., "A network flow algorithm for reconstructing binary images from discrete x-rays," Journal of Mathematical Imaging and Vision 27(2), 175-191 (2007).

[18] Myers, G., Paganin, D., Gureyev, T., and Mayo, S., "Phase-contrast tomography of single-material objects from few projections," Optics Express 16(2), 908-919 (2008).

[19] Kuba, A., "Reconstruction of two-directionally connected binary patterns from their two orthogonal projections," Computer Vision, Graphics, and Image Processing 27, 249-265 (1984).

[20] Kingston, A., Parrein, B., and Autrusseau, F., "Redundant image representation via multi-scale digital Radon projections," in [to be presented at Int. Conf. on Image Processing (ICIP)], (2008). 